\title{
Transalpina
}

\section{Le anime semplici e il piedistallo : l'immagine del Duce condottiero nei libri scolastici e per ragazzi dell'Italia fascista}

\section{Beatrice Sica}

\section{OpenEdition}

\section{Journals}

\section{Edizione digitale}

URL: http://journals.openedition.org/transalpina/314

DOI: $10.4000 /$ transalpina.314

ISSN: 2534-5184

\section{Editore}

Presses universitaires de Caen

\section{Edizione cartacea}

Data di pubblicazione: 1 octobre 2018

Paginazione: 191-214

ISBN: 978-2-84133-900-6

ISSN: 1278-334X

\section{Notizia bibliografica digitale}

Beatrice Sica, «Le anime semplici e il piedistallo : l'immagine del Duce condottiero nei libri scolastici e per ragazzi dell'Italia fascista », Transalpina [Online], 21 | 2018, online dal 19 décembre 2019, consultato il 21 décembre 2020. URL : http://journals.openedition.org/transalpina/314 ; DOI : https:// doi.org/10.4000/transalpina.314 


\title{
LE ANIME SEMPLICI E IL PIEDISTALLO : L'IMMAGINE DEL DUCE CONDOTTIERO NEI LIBRI SCOLASTICI E PER RAGAZZI DELL'ITALIA FASCISTA ${ }^{1}$
}

\begin{abstract}
Riassunto: L'articolo esamina la rappresentazione di Mussolini a cavallo nel mondo dell'editoria scolastica e per ragazzi durante il regime fascista. Sono presi in considerazione sia testi che immagini, mettendo in luce lo scambio che si attuava tra il mondo dei bambini e quello degli adulti soprattutto attraverso fotografie, cartoline, grafica. Attraverso la nozione di performativity già mutuata da Judith Butler in studi sulla costruzione dell'identità nazionale dei bambini nei regimi totalitari l'autrice mostra come l'icona del Duce condottiero non fosse soltanto subìta dai fanciulli ma implicasse una loro partecipazione attiva che li coinvolgeva in maniera ancora più profonda nel sistema di valori su cui era basata la dittatura mussoliniana.
\end{abstract}

Résumé: L'article examine la représentation de Mussolini à cheval dans le monde de l'édition pour les écoles et pour la jeunesse pendant le fascisme; s'appuyant sur des textes en prose et en vers, des photos, des cartes postales et des illustrations, il met en évidence les échanges de mots et d'images qui avaient lieu entre le monde des enfants et celui des adultes. À travers la notion de performativity de Judith Butler, déjà empruntée dans des études sur la construction de l'identité nationale des enfants sous les régimes totalitaires, l'autrice montre comment l'icône du Duce condottiere, loin d'être seulement subie par les enfants, comportait une participation active qui les impliquait encore plus profondément dans le système de valeurs sur lequel était fondée la dictature mussolinienne.

Nel novembre del 1937 il Centro Sperimentale di Cinematografia di Roma organizzò una proiezione del film Scipione l'Africano di Carmine Gallone nella scuola elementare Federico Di Donato della capitale. Dopo la proiezione fu chiesto agli scolari di terza, quarta e quinta di esprimere le loro impressioni su quello che avevano visto; i loro temi e disegni furono pubblicati nell'agosto del 1939 in un numero speciale della rivista

1. The publication of this article had the support of the Istituto di Studi Avanzati of the University of Bologna, the EURIAS Fellowship Programme and the European Commission (Marie Sklodowska-Curie Actions - COFUND Programme - FP7). 
Bianco e Nero ${ }^{2}$. Nella premessa a quel numero speciale Luigi Chiarini, direttore del Centro e della rivista, assicurava che temi e disegni erano stati prodotti «immediatamente dopo la visione del film» e che rispecchiavano il pensiero degli scolari «senza che i maestri» li avessero «orientati» o avessero "suggerito delle idee $»^{3}$. Anche se un'influenza degli adulti è comunque ravvisabile ${ }^{4}$, la notazione interessa per il presupposto a cui si accompagnava. Scriveva infatti Chiarini :

La psicologia infantile, semplice e spontanea, si avvicina molto a quella delle masse più ingenue. [...] Ecco la ragione per cui Scipione l'Africano è stato portato ai bambini: si voleva constatare se nelle anime più semplici i risultati propostisi erano stati raggiunti, cioè se il film piaceva, se accendeva gli spiriti alla rievocazione della grandezza di Roma, se faceva pensare al riallacciarsi del Fascismo a questa più grande nostra tradizione ${ }^{5}$.

Come ha scritto Antonio Gibelli, «che il pubblico bambino sia inteso quale modello e metafora dell'intero popolo italiano, cui proporre le gesta del regime come un racconto avventuroso, fascinoso e facilmente assimilabile, è evidente ${ }^{6}$, anche qui dove quelle gesta non sono raccontate direttamente ma attraverso una trasposizione storica. Neanche a farlo apposta, l'esperimento di Chiarini poteva dirsi perfettamente riuscito: i temi degli scolari sono pieni di parallelismi tra la Roma antica e quella fascista, tra la battaglia di Canne e la sconfitta di Adua, tra la riscossa di Zama e la conquista dell'Etiopia, tra l'entusiasmo del popolo romano antico per Scipione e quello degli italiani per il Duce, e soprattutto tra la figura di Scipione e Mussolini. Nota ancora Chiarini :

Le anime più semplici si sono trovate di fronte a uno Scipione e ad un Annibale più vicini alla loro immaginazione, a quella immaginazione che fa considerare gli eroi del passato, un po' togati ed aulici, e non come uomini di tutti i giorni $[\ldots]$. Certo uno Scipione $[\ldots]$, diciamo così, più sulla terra al

2. Bianco e nero, III, 8, agosto 1939. Il nome della scuola si ricava da p. 65.

3. L. Chiarini, «Premessa», ibid., p. 10.

4. Lo si nota per esempio in alcune considerazioni che evidentemente i bambini avevano sentito fare dagli adulti e che sono ricorrenti, come quella a proposito della competizione con il contemporaneo cinema americano. Quasi tutti inoltre si soffermano sulla scena finale del film, in cui Scipione infilando la mano in un grande sacco di grano che un servo gli porge, esclama: «Buon grano. E domani con l'aiuto degli dei comincerà la semina». Di questa scena il bambino Mario Borello scrive: "Che avrà voluto significare? Non sarei riuscito a comprenderlo bene, se il Sig. Maestro non ce lo avesse spiegato" (Bianco e nero, p. 64).

5. L. Chiarini, «Premessa», p. 9-10.

6. A. Gibelli, Il popolo bambino. Infanzia e nazione dalla Grande Guerra a Salò, Torino, Einaudi, 2005, p. 232. 
livello degli altri e meno su un piedestallo, sarebbe stato più vero, ma certo meno vicino alla fantasia popolare della quale i bambini sono tanta parte. Significativa, sotto questo aspetto, [...] l'osservazione della piccola Lucia Priami: «Era bello Scipione sul suo cavallo bianco. Egli fissava i Romani [...] con gesto forte e animatore, e pareva dicesse: dobbiamo vincere ad ogni costo! Proprio come fa oggi il nostro amato Duce quando parla ai nostri valorosi soldati. [...]». Da essa è chiaro come l'anima popolare ponga l'eroe immediatamente su un piedestallo e in un'atmosfera di leggenda, tale da arrivare addirittura a delle trasposizioni $[\ldots]^{7}$.

L'equazione di Chiarini tra bambini e popolo non era nuova, ma si riallacciava ai presupposti della pedagogia di ispirazione borghese che aveva animato l'Italia fin dal periodo post-unitario ${ }^{8}$. Il regime di Mussolini, però, sorto dopo l'esperienza della Grande Guerra che aveva visto la prima vera esperienza di nazionalizzazione dell'infanzia ${ }^{9}$, poneva il militarismo al centro del processo educativo. Come ha scritto Umberto Silva, «L'interesse del fascismo per la gioventù [...] aveva uno scopo ben preciso: rivendicare in maniera totalitaria e autoritaria l'educazione del cittadino, imporre alla gioventù il senso della virilità, della potenza, della conquista $»^{10}$.

Gli storici hanno già ampiamente analizzato le diverse forme e i vari canali attraverso cui fu proposta e imposta al cittadino, e quindi in primis

7. L. Chiarini, «Premessa», p. 11-12.

8. Cfr. M. Bacigalupi, P. Fossati, Da plebe a popolo. L'educazione popolare nei libri di scuola dall'Unità d'Italia alla Repubblica, Scandicci (FI), La Nuova Italia, 1986, p. 6-7: «La borghesia risorgimentale sentiva l'urgenza di conquistare il popolo non tanto a progetti politici di cui lei sola riteneva di potere e dovere sopportare il peso quanto ai propositi di rinascita economica e di conquista di quel grado di "civiltà" considerato indispensabile alla fondazione morale del nuovo stato. Più tardi, nei primi decenni dopo l'Unità, la parte più dinamica e di più larghe vedute della classe dirigente si pose il problema di fondare sulla trasmissione di una serie di comportamenti ispirati ai valori centrali del lavoro e della patria, l'unificazione reale e lo sviluppo industriale del paese. [...] in epoca risorgimentale, quella schiera di intellettuali che aderirono alla politica del moderatismo liberale e che provenivano in gran parte dal ceto medio [...] attribuirono alla propria posizione sociale una funzione mediatrice tra élites e masse popolari che fece della classe media il modello cui il popolo doveva guardare e contribuì a fissare molte costanti della nuova etica proposta al popolo: moderazione, rispettabilità, equilibrio tra l'aspirazione a migliorarsi e la capacità di contentarsi di ciò che si ha. Questi modelli passarono attraverso una copiosa produzione di giornali, opuscoli divulgativi, testi educativi che divenne in seguito editoria specificamente scolastica».

9. Cfr. A. Gibelli, Il popolo bambino..., p. 20 : «L'idea di uno Stato che accompagna i nuovi nati dalla culla alla divisa, alla trincea e se necessario alla tomba $[\ldots]$ nasce nel contesto della Grande Guerra [...]. Molti elementi del rituale, della gestualità che saranno dei balilla mussoliniani compaiono occasionalmente prima che il fascismo se ne appropri».

10. U. Silva, Ideologia e arte del fascismo, Milano, Mazzotta, 1973, p. 49. 
ai bambini, questa educazione da parte del regime, soffermandosi anche sul mondo dell'editoria scolastica e per ragazzi ${ }^{11}$. Qualcosa però si può forse ancora dire a proposito di quell'immagine precisa di Mussolini a cavallo che la piccola Lucia Priami, come tanti altri bambini cresciuti nell'Italia fascista, sovrapponeva a quella di Scipione e in cui Chiarini ravvisava particolarmente l'attitudine eroicizzante tipica della "fantasia popolare». Perché gli scolari pensavano al Duce quando sullo schermo vedevano l'attore Annibale Ninchi alto in sella interpretare Scipione che incitava i suoi legionari? Se il mondo della scuola funzionava da laboratorio della più vasta opera di educazione nazionale, come veniva proposta l'immagine del Duce a cavallo nei testi scolastici e nella letteratura per bambini e ragazzi ? E quale tipo di osmosi si creava tra il mondo della scuola e l'universo degli adulti ? Era così «semplice e spontanea» come voleva Chiarini l'attitudine eroicizzante con cui veniva recepita quell'immagine?

\section{Fotografie, cartoline, grafica e affreschi}

Le fotografie e le cartoline furono tra i mezzi attraverso cui l'immagine del Duce si diffuse più ampiamente nell'Italia fascista ${ }^{12}$. Tra le tante prodotte dal regime numerose erano quelle che ritraevano Mussolini a cavallo: era il suo ritratto ufficiale, che circolava sia ai livelli più alti che a quelli più

11. Si vedano almeno M. Bacigalupi, P. Fossati, Da plebe a popolo..., in particolare il capitolo «Alla ricerca del testo fascista» (p. 152-187) e «I testi unici di Stato» (p. 191-231); A. Ascenzi e R. Sani, Il libro per la scuola tra idealismo e fascismo. L'opera della Commissione centrale per l'esame dei libri di testo da Giuseppe Lombardo Radice ad Alessandro Melchiori (1923-1928), Milano, Vita e Pensiero, 2005; M. Galfrè, Il regime degli editori. Libri, scuola e fascismo, Roma - Bari, Laterza, 2005; M. Colin, «Les livres de lecture italiens pour l'école primaire sous le fascisme (1923-1943)", Histoire de l'éducation, 127, juillet-septembre 2010, p. 57-94; M. Colin, I bambini di Mussolini. Letteratura, libri, letture per l'infanzia sotto il fascismo, Brescia, La Scuola, 2012 ; Piccoli eroi. Libri e scrittori per ragazzi durante il ventennio fascista, M. Castoldi (a cura di), Milano, Franco Angeli, 2016.

12. Sull'uso della fotografia come strumento di propaganda dell'immagine del Duce, cfr. almeno S. Falasca-Zamponi, Lo spettacolo del fascismo, Soveria Mannelli, Rubbettino, 2003, soprattutto le p. 85-88, 127-129, 133 e in particolare per le immagini di Mussolini a cavallo le p. 114-115; S. Luzzatto, L'immagine del Duce. Mussolini nelle fotografie dell'Istituto Luce, Roma, Editori Riuniti, 2001, che nell'Introduzione alle p. 8-9 fa anche alcune considerazioni in particolare su Mussolini a cavallo; e A. Antola, "Photographing Mussolini», in The Cult of the Duce. Mussolini and the Italians, S. Gundle, C. Duggan and G. Pieri (a cura di), Manchester, Manchester University Press, 2013, p. 178-192. Sulle cartoline cfr. E. Sturani, Otto milioni di cartoline per il Duce, Torino, Centro Scientifico Editore, 1995, rielaborato poi in Le cartoline per il Duce, Torino, Edizioni del Capricorno, 2002; dello stesso, in forma più breve, «Il fascismo in cartolina», in Modernità totalitaria. Il fascismo italiano, E. Gentile (a cura di), Roma - Bari, Laterza, 2008, p. 112-128, e «Analysing Mussolini postcards», Modern Italy, XVIII, 2, 2013, p. 141-156. 
bassi della compagine sociale. Il capo del Fascismo amava presentarsi in tenuta da cavallerizzo o regalare foto di sé a cavallo ai visitatori stranieri; d'altra parte i bambini collezionavano foto e cartoline di lui sugli arcioni e le portavano a scuola ai maestri ${ }^{13}$. E se non capitavano loro fra le mani per altre vie, i fanciulli in età scolare trovavano quei ritratti equestri del Duce nei libri di testo o sui quaderni di scuola.

La foto scattata da Eugenio Risi all'ippodromo di villa Glori a Roma il 30 ottobre 1927 in occasione dell'annuale della marcia su Roma (fig. 1) divenne prima una cartolina che ritraeva Mussolini e Augusto Turati insieme; poi, nuovamente ritoccata, una cartolina dove soltanto la figura di Mussolini a cavallo con il braccio destro alzato campeggiava su sfondo uniforme. In quest'ultima versione l'immagine passò anche nei libri di scuola. La si ritrova per esempio nel Libro della seconda classe del 1930 (fig. 2) a illustrare una lettura intitolata «Bandiere alle finestre» che celebra la ricorrenza del 28 ottobre nella forma di un dialogo tra la maestra e gli scolari entusiasti e attivamente partecipi:

$-[\ldots]$ domani tutti i balconi e tutte le finestre saranno imbandierati. E sapete perché?

- Sissignora! - gridarono i bambini balzando in piedi.

- Bravi! [...] che giorno è domani?

- Il 28 ottobre.

- E che cosa è il «ventotto ottobre»?

- È l'anniversario della Marcia su Roma.

— [...] Badate, ragazzi: anche se doveste fabbricarla con la carta, domani ogni finestra dovrà avere la sua bandiera.

- Sissignora! Eia, eia, eia, alalà ${ }^{14}$ !

13. Cfr. L. Passerini, Mussolini immaginario, Roma - Bari, Laterza, 1991, p. 198, che racconta della giornalista Paule Herfort in visita a Mussolini per la seconda volta : «Mussolini, vestito da cavallerizzo, l'aveva guardata in faccia, il mento alto con gesto di sfida» etc. In bibliografia Passerini rimanda a P. Herfort, Chez les romains fascistes, Parigi, Éditions de la «Revue mondiale», 1934. Cfr. anche P. Carlson, «Naivete abroad. Will Rogers goes mano a mano with Mussolini», American History, LI, 3, August 2016, p. 15: "After a half-hour, Mussolini called an aide, who scurried off, returning with a photo of Il Duce on horseback the dictator autographed for Rogers». Sui bambini che collezionavano immagini di Mussolini a cavallo cfr. D. Mingozzi, Mussolini visto dai ragazzi, con prefazione di Augusto Turati, San Casciano Val di Pesa (Firenze), Società Editrice Toscana, 1928, p. 101: «è tutta una serie di fotografie che mi sciorinano sotto gli occhi. Mussolini, in piedi, a cavallo, seduto alla scrivania, in abito militare, ministeriale, diplomatico ».

14. Il libro della seconda classe, compilato dalla signora O. Quercia Tanzarella (Ornella), illustrato da M. Pompei, Roma, Libreria dello Stato, 1930, p. 21 e 23 ; la foto di Mussolini a cavallo è a p. 22. 
La stessa fotografia si trova anche nell'edizione del 1934, questa volta ad accompagnare una lettura intitolata «Il bimbo prodigioso (il Duce racconta)» in cui vengono narrate le vicende del Capo del Fascismo in prima persona ${ }^{15}$.

Un'altra foto ( $f i g .3$ ), scattata a Roma il 24 maggio 1934 e diventata probabilmente anch'essa una cartolina, fu usata per illustrare il retro di una serie di quaderni stampati dalle cartiere Donzelli di Milano: qui il Duce a cavallo, incastonato in un tondo, era accompagnato da una stella soprastante, dal fascio littorio sulla sinistra e da una scritta posta in un riquadro sottostante che variava a seconda dei quaderni ${ }^{16}$.

Le fotografie che venivano ristampate direttamente, cartolinizzate o riprodotte graficamente non erano il solo veicolo per la diffusione dell'immagine di Mussolini a cavallo nel mondo della scuola. Come ha scritto Luisa Passerini, «è da notare [... la grande importanza della grafica, in particolare nella letteratura per i ragazzi, scolastica e non : la duttilità del mezzo espressivo gli conferisce straordinarie capacità di esaltare l'immaginazione ${ }^{17}$. Così si potevano trovare dei veri e propri ritratti equestri che si ispiravano non alle foto coeve ma, per esempio, a opere d'arte del passato. Nel libro L'aratro e la spada. Letture per la terza classe dei centri rurali del 1941 si trova Mussolini a cavallo in un disegno di Pio Pullini (fig. 4) ${ }^{18}$. Pullini ritrae Mussolini di lato incorniciando la scena entro un arco a tutto sesto. Dietro al Duce e al suo cavallo bianco si intravvedono dei legionari, anch'essi raffigurati di lato, che marciano serrati a fianco del Duce innalzando moschetti e stendardi. Le linee delle gambe dei legionari sotto la pancia del cavallo e quelle dei moschetti e degli stendardi sopra il dorso sono perfettamente diritte e allineate tra loro e si incrociano convergendo al centro dove campeggia in primo piano il Duce in sella. Le figure sono appiattite, non c'è profondità in questo spazio, ma cavallo, cavaliere e soldati sullo sfondo sono un blocco compatto di linee. Si tratta di un disegno molto raffinato dal punto di vista della composizione: la visione laterale, l'incorniciatura della scena, la costruzione delle figure sono ispirate alla migliore tradizione artistica italiana. È evidente che qui Pullini si richiama ai monumenti equestri di Niccolò da Tolentino e di Giovanni Acuto affrescati da Andrea del Castagno e da Paolo Uccello nel Duomo di Firenze.

15. Nell'edizione del 1934 la foto di Mussolini a cavallo è a p. 32.

16. Uno di questi quaderni si può vedere in L. Marrella, I quaderni del Duce. Tra immagine e parola, Manduria, Barbieri, 1995, p. 57.

17. L. Passerini, Mussolini immaginario, p. 203.

18. L'aratro e la spada. Letture per la terza classe dei centri rurali, testo di A. Petrucci, illustrazioni di P. Pullini, Roma, Libreria dello Stato, 1941, p. 192. 
Nello stesso libro di scuola c'è anche una lettura di Alfredo Petrucci intitolata «Il pittore ha finito", in cui si immagina un vero e proprio affresco con Mussolini condottiero:

Il pittore ha finito di decorare la sala delle adunanze nella Casa del Fascio, dipingendo per ultime la parete sulla porta d'ingresso e quella dirimpetto. Sulla prima egli ha rievocato la Marcia su Roma: il Duce a cavallo, seguito dai suoi intrepidi legionari, marcia verso la Città Eterna, che si vede nello sfondo, con le sue cupole, le sue torri, i suoi palazzi monumentali. E da tutto il dipinto emana un senso di fede, di ardore, di risolutezza: la fede l'ardore, la risolutezza che portano dritto alla vittoria.

- Ah, - dice Orazio fra sé e sé - saper dipingere così!

Sul dipinto della parete dirimpetto il pittore ha rappresentato la proclamazione dell'Impero fascista. [...]

Orazio, pieno di ammirazione, guarda, osserva, riflette, e appena tornato a casa impugna le sue matitine colorate e comincia a stropicciarle sulla carta. Vuol rifare i due dipinti ${ }^{19}$.

Il brano di Petrucci rispecchia una situazione comune in Italia negli anni del regime: soprattutto dalla seconda metà degli anni Trenta, dalla proclamazione dell'Impero in avanti, molti artisti vennero chiamati a decorare palazzi ed edifici pubblici con raffigurazioni del Duce a cavallo nelle vesti di fondatore dell'Impero fascista, oppure, come in questa lettura, al tempo della marcia su Roma (con evidente stravolgimento e trasfigurazione della realtà storica, visto che Mussolini non era a cavallo il 28 ottobre del 1922, bensì si recò nella capitale in treno). Il Duce condottiero a cavallo in marcia verso la Città Eterna si vede per esempio nell'affresco di Mario Capuzzo recentemente riportato alla luce sullo scalone di palazzo Koch, sede della Carife in corso Giovecca a Ferrara, oppure si poteva vedere nel mosaico realizzato su disegno di Anton Giuseppe Santagata nella Casa del Mutilato di Ravenna, oggi distrutto ${ }^{20}$. Più che per la rispondenza ideale con opere coeve, però, il brano di Petrucci interessa qui per i significati

19. A. Petrucci, «Il pittore ha finito», in L'aratro e la spada..., p. 32.

20. Un'immagine dell'affresco di Capuzzo si può vedere in G. Inzerillo, «La memoria del passato", Ferrara. Voci di una città, "Rivista semestrale di cultura, informazione e attualità della Fondazione Cassa di Risparmio di Ferrara», VI, 14 (2001), consultabile su http://rivista.fondazionecarife.it/it/luoghi/item/232-la-memoria-del-passato/232-lamemoria-del-passato (accesso effettuato il 26 maggio 2018). Il bozzetto per il mosaico oggi distrutto che era nella Casa del Mutilato di Ravenna si può vedere in La nuova Casa del Mutilato di Ravenna. Storia, arte, architettura, I. Simonini (a cura di), Ravenna, Edizioni del Girasole, 2002, p. 96 ; per le notizie sul mosaico si veda V. Sgarbi, «La rinascita del mosaico italiano nell'epoca fascista», ibid., p. 79-80. 
che associa all'immagine del Duce a cavallo: fede, ardore, risolutezza, sentimento della vittoria. È da notare l'atteggiamento del piccolo Orazio nel racconto : «pieno di ammirazione, guarda, osserva, riflette » e una volta a casa si ingegna per riprodurre l'opera, spinto evidentemente dall'esaltazione - dalla fede, dall'ardore, dalla risolutezza, dal sentimento della vittoria - che essa suscita.

\section{Poesie, disegni, ricordi e sogni}

Il Duce a cavallo si ritrova anche in visioni e sogni dei bambini raccontati in versi e in prosa. Nel libro L'aratro e la spada menzionato prima, proprio sotto il disegno di Pullini, si trova una poesia di Alfredo Petrucci intitolata «Il Duce», di cui citeremo qui soltanto i primi tre distici: «Cavalca, e sotto il suo passo / sprizza scintille il sasso. / Lo seguon le ferree legioni / e il mondo par che ne tuoni. / L'Italia grande, l'Impero: / questo fu il suo pensiero $»^{21}$. I versi di Petrucci sono molto meno raffinati del disegno di Pullini che li accompagna, ma interessano ugualmente qui per i significati che associano alla figura del Duce condottiero : l'energia (il sasso che «sprizza scintille» al passaggio del cavaliere); la forza («le ferree legioni» che seguono il capo); la potenza (la sensazione che il mondo «tuoni» al passare degli eserciti del condottiero); il prestigio nazionale («L'Italia grande») anche sul piano politico («l'Impero»).

Un'altra poesia con Mussolini a cavallo si trova nel Libro della terza classe elementare del 1936; è di Nazareno Padellaro ed è intitolata «Il Duce e il bambino ». Delle cinque strofe citiamo qui soltanto la prima e le ultime due:

Tu levi la piccola mano

col viso di luce irradiato.

Tu sei quel bambino italiano,

che il Duce a cavallo, ha incontrato.

$[\ldots]$

«Ti guardo, bambino. La fede, che il cuore mi fa come il ferro, s'accende più viva e più crede. Più forte il destino a me serro.

21. Cfr. n. 18 
«Io lotto e combatto per te, o piccolo dolce italiano.

E palpita il sogno ch'è in me, per questo saluto romano ${ }^{22}$.

Se nel brano in prosa di Petrucci visto sopra il piccolo Orazio si esalta di fronte a un dipinto con il Duce a cavallo e la sua eccitazione si esprime nel tentativo di emulare a casa il pittore, in questi versi di Padellaro il bambino - identificato con un «tu» generico in cui ogni piccolo lettore poteva ancor più facilmente rispecchiarsi - incontra invece il Duce a cavallo in persona. Mussolini stesso parla rispondendo al saluto romano del fanciullo, in un'atmosfera permeata di fede fascista, impegno militare, fiducia nel futuro glorioso. «Io lotto e combatto per te», dichiara il Duce condottiero nell'ultima strofa: così il bambino, con il suo saluto romano, diventa agente attivo del sentimento nazionale, dello spirito guerriero fascista e dei destini della patria.

Il brano di Petrucci «Il pittore ha finito » è di ambientazione realistica. Questa narrazione in versi di Padellaro si svolge in un'atmosfera luminosa, irreale, quasi mistica. Più smaccatamente tendente al favolistico-avventuroso, o a quel genere che Mariella Colin ha definito «meraviglioso fascista ${ }^{23}$, è invece il libro per ragazzi di Paolo Alberto Colombini, Filo e Refe alla scoperta di un tesoro (1934) ${ }^{24}$, dove pure c'è un incontro con il Duce a cavallo (fig. 5). In questo racconto, come sintetizza M. Colin, «Filo e Refe sono due Balilla svogliati, che non conoscono nemmeno le parole dell'inno fascista Giovinezza; ragion per cui vengono puniti dal loro caposquadriglia. Nella notte, Filo sogna che Mussolini gli appare sotto le sembianze di un principe ${ }^{25}$ a cavallo, svelandogli l'esistenza di un tesoro nascosto nel deserto di Tripoli. Ecco la descrizione del Duce sul suo destriero che si trova nel secondo capitolo, intitolato «Un sogno meraviglioso». Filo racconta a Refe:

Ho fatto un sogno meraviglioso. [...] Ho sognato il Duce [...] sissignore, il Duce in persona. Ero in un grandissimo prato, in una luminosa spianata... c'erano tante legioni di Balilla in divisa, moschettieri, esploratori escursionisti... si aspettava la visita del Duce che doveva passarci tutti in rivista... [...] si ode finalmente uno squillo di tromba, che annunzia l'arrivo del Duce. Infatti, dopo

22. Il libro della terza classe elementare, testo di N. Padellaro, illustrazioni di C. Testi, Roma, Libreria dello Stato, 1936, p. 189.

23. M. Colin, I bambini di Mussolini..., p. 298.

24. P.A. Colombini, Filo e Refe alla scoperta di un tesoro, Milano, Istituto Tipografico Editoriale, 1934 .

25. M. Colin, I bambini di Mussolini..., p. 298. 
poco, lo vediamo spuntare da un nuvolo di polvere bianca come l'ovatta... [...] Poi udiamo avvicinarsi lo scalpitio di cento cavalli focosi... Il Duce è davanti a tutti e cavalca un cavallo tutto d'argento, con gli zoccoli d'oro, con la criniera e la coda di fiamma, con le narici di fuoco e gli occhi di diamanti... [...] vestito da Comandante Supremo, porta in capo un elmo di madreperla, e sull'elmo un pennacchio bianco come il latte... [...] passa davanti alle legioni dei Balilla e sorride ai più piccini... [...] Allora [...] avviene un fatto... [...] Avviene che il Duce, giunto davanti a me, ferma il suo cavallo d'argento, con gli zoccoli d'oro, con la criniera e la coda di fiamma, con le narici di fuoco e gli occhi di diamanti, e mi guarda... [...] Poi il Duce mi fa un cenno con la mano e mi chiama per nome... [...] io saluto romanamente... ma egli mi vuole vicino a sé. [...] si sporge dall'arcione, e col suo braccio di ferro mi solleva da terra, mi porta vicino a sé e mi dice in un orecchio... [...] «Tu sei un bravo Balilla... e io ti voglio ricompensare... Come ricompensa, ti svelo dove si trova un grande tesoro... [...]». [...] Poi mi ha baciato, ha spronato il suo cavallo, e al galoppo, è scomparso in mezzo ad un'altra nuvola bianca come l'ovatta... ${ }^{26}$.

La scena ripropone qualcosa che i bambini nell'Italia fascista conoscevano bene: la rivista delle forze armate, cui partecipavano anche le squadre di Balilla e Piccole Italiane. Sull'importanza di queste parate e adunate nel rituale fascista è già stato scritto ${ }^{27}$; per quanto riguarda la parte in cui intervenivano i bambini, possiamo farci un'idea della coreografia che interessava i più piccoli guardando i filmati e le foto (per es. la fig. 6) conservati nell'archivio storico dell'Istituto Luce ${ }^{28}$. Per i bambini sfilare di fronte a Mussolini a cavallo o

26. P.A. Colombini, Filo e Refe alla scoperta di un tesoro, p. 20-23.

27. Qui basterà rimandare $\mathrm{a}$ E. Gentile, Il culto del littorio, Roma - Bari, Laterza, 2009, soprattutto il capitolo IV, Liturgia dell' "armonico collettivo», p. 139-174.

28. I filmati e le fotografie dell'archivio dell'Istituto Luce sono visibili sul sito internet http:// www.archivioluce.com/archivio (accesso effettuato il 26 maggio 2018). Tra i filmati più eloquenti è certamente il Giornale Luce B/Bo332 (codice filmato: Bo33204), «Roma. La visita del Capo del governo al Campo Dux", in particolare le sequenze con minutaggio interno al filmato da 00:17:21:15:04 a 00:18:16:08:04 (corrispondenti nel video caricato su YouTube al tempo da 1:19 a 2:13); ma si vedano anche: Giornale Luce A/Ao32o (codice filmato: A032001), "A Roma si celebra il 21 aprile», in particolare le sequenze con minutaggio interno al filmato da o0:42:27:03 a o0:44:06:06 e da 00:44:20:03 a $00: 44: 25: 09$ (YouTube da 3:39 a 4:18 e da 5:32 a 5:37); Giornale Luce B/Bo319 (codice filmato: Bo31906), «La palestra della nazione», in particolare le sequenze con minutaggio interno al filmato da 00:41:05:18:03 a 00:41:18:10:04 (YouTube da 1:39 a 1:52); Giornale Luce B7Bo476 (codice filmato: Bo47604), "Celebrazione della Ottava Leva fascista», in particolare le sequenze con minutaggio interno al filmato da o0:02:23:03:02 a 00:03:29:04:01 e da oo:03:48:24:04 a 00:04:11:11:02 (YouTube da 0:04 a 1:11 e da 1:30 a 1:52); Giornale Luce B/Bo892 (codice filmato: Bo89204), «Annuale dell'intervento celebrato con la X leva fascista alla presenza di Mussolini», in particolare le sequenze con minutaggio interno al 
vederlo alto in sella che li passava in rassegna mentre erano schierati sull'attenti era certamente un momento solenne e importante che si imprimeva nella memoria: lo raccontavano infatti nei loro temi e lo disegnavano (per es. fig. 7), come si ricava dal libro di Dolores Mingozzi Mussolini visto dai ragazzi $(1928)^{29}$; e finivano anche per sognarlo ad occhi aperti, stando a quanto racconta Franco Ciarlantini nel suo libro Mussolini immaginario (1933):

L'immagine fantastica che tanti si fanno di Mussolini entra, come la fiaba, nel sogno dei fanciulli. [...] L'immagine di Mussolini a cavallo ricorre continuamente.

Di bimbi, come il piccolo Luigi Arienti di Legnano che scrivono di lui cose di questo genere, ce ne sono un'infinità:

«Nella mia fantasia vedo spesso il Duce a cavallo, colla testa alta, coll'occhio scrutatore in divisa di parata, passare in rivista le truppe che gridano: - A noi! - Egli guarda severo come per dire: - Ricordatevi che questo grido vi impegna a seguirmi dove io vi condurrò, $\mathrm{o}$ in pace o in guerra».

[...] Rina Giussanti torna all'idea del Condottiero ferrigno:

«Il Duce come Capo del Fascismo, quando passa fiero nella divisa nera, sul suo cavallo farà soggezione a tutti. E quando passerà in rivista la Milizia fascista, i Balilla e le Piccole Italiane, tutti alzeranno il braccio a lui urlando: - A noi! - Ed Egli li guarderà con viso duro come per dire: - Non ci vogliono chiacchere, ma fatti! $»^{30}$.

Rispetto al senso di disciplina restituito dalle parole di Luigi Arienti e di Rina Giussanti riportate da Ciarlantini, il brano di Colombini visto sopra trasfigura la rivista delle forze armate e stempera l'atmosfera militaresca nella leggerezza di una favola. Nel racconto di Filo il Duce è come un principe buono che soccorre i due piccoli birichini e svogliati. La rappresentazione favolistica di Colombini è di stampo popolare: quel cavallo d'argento con zoccoli d'oro, criniera e coda di fiamma, narici di fuoco e occhi di diamanti - elementi ripetuti nel testo come in una narrazione di tipo orale - e il Duce con l'elmo di madreperla e il pennacchio bianco come il latte rappresentano un campionario di materiali pregevoli ed elementi straordinari che qui sfiora

filmato da 00:13:41:10:03 a 00:13:14:04:02 (YouTube da 1:00 a 1:32). Tra le fotografie, sono da segnalare quelle con con segnatura Aooo49911, Aooo49912, Aooo49913, Aooo49914 (Campo Dux), Aoo054560, Aoo054570, Aoo054582 (ottava leva fascista) e Aooo64869, Aooo64875, Aooo64879, Aooo64880, Aooo64888 (decima leva fascista).

29. Dolores Mingozzi riporta i temi e disegni con cui i bambini di varie scuole d'Italia raccontarono come vedevano il Duce. Per i temi dove Mussolini è menzionato a cavallo, cfr. le p. 21, 27, 30, 32, 43, 46, 81, 88, 101, 141; per i disegni che lo ritraggono in sella, le p. $21,23,24,27,30,32,33,34,90$. La fig. 7 qui è tratta da p. 43.

30. F. Ciarlantini, Mussolini immaginario, Milano, Sonzogno, 1933, p. 162-163, 181-182. 
addirittura il kitsch. Siamo di fronte a un vero e proprio simulacro di tipo devozionale, che però non suscita ammirazione statica ma innesca tutta l'azione. Prescelto dal Duce come depositario del segreto del tesoro, infatti, Filo parte con il fratello Refe da Milano alla volta di Tripoli su un pallone aerostatico di casalinga fattura. Arrivati nel deserto i due fratelli recuperano il tesoro (un piccolo Balilla meccanico che ogni volta che viene caricato a molla canta l'inno fascista Giovinezza), uccidono le belve feroci che minacciano l'oasi lì vicino e vengono incoronati re dalla tribù locale di indigeni. I due Balilla nominano subito un Ministro della Guerra indigeno e impartiscono ordini precisi : insegnare l'inno fascista a tutti $\mathrm{i}$ «bambini negri», «fare la Leva Fascista di tutti i bambini selvaggi, dai sei ai quattordici anni $»^{31}$ e insegnare loro il saluto romano. La cerimonia dell'incoronazione di Filo e Refe ricalca da vicino le parate dell'Italia fascista ma i due piccoli protagonisti, nonostante la nomina regale, si premurano di non attribuire a se stessi nessun merito:

Noi non siamo che due umili e piccoli soldati della nuova Italia [...]; siamo Balilla di quell'Italia sulla quale governa l'Uomo più grande del nostro secolo: Benito Mussolini, Duce del Fascismo Italiano e, fra non molto, Duce del Fascismo del mondo ${ }^{32}$ !

Alla fine del racconto di Colombini scopriamo che questa avventura è stata solo in sogno: un sogno che ha permesso, però, a Filo e Refe di imparare finalmente le parole dell'inno fascista Giovinezza che la mattina, svegliati come al solito dalla madre, i due bambini sono in grado di cantare alla perfezione conoscendone finalmente i versi a memoria.

Un altro sogno di Balilla al servizio dell' "Uomo a cavallo » e della sua potenza nel mondo si trova a conclusione del libro scolastico L'aratro e la spada già menzionato, in una lettura che racconta di un campo Balilla con tanti bambini che dopo le attività giornaliere si addormentano stanchi sognando ognuno qualcosa. Ecco come Petrucci conclude il brano e con esso l'intero volume:

Leonetto invece crede di essersi arrampicato su Monte Sacro, e lì in cima, col suo tamburo a tracolla, suona suona suona; suona tanto da farsi udire da tutti, in qualunque parte del globo si trovino, e sveglia i dormienti, sferza i pigri, mentre giù al piano un Uomo a cavallo, seguito da una fiumana di popolo in camicia nera, s'incammina verso il sole ${ }^{33}$.

31. P.A. Colombini, Filo e Refe alla scoperta di un tesoro, p. 129 e 130.

32. Ibid., p. 148.

33. A. Petrucci, «Siam Balilla», in L'aratro e la spada..., p. 210. 
A illustrazione di questo brano si trova nell'ultima pagina del libro un altro disegno di Pio Pullini (fig. 8), questa volta con il Balilla in primo piano: Leonetto, ritratto di schiena leggermente volto verso sinistra, si erge con il suo tamburo a tracolla su un'altura (il Monte Sacro) dalla quale vede - e noi con lui - in basso, al piano, sempre di schiena, il Duce a cavallo con il braccio destro alzato e una moltitudine di camicie nere che lo seguono a piedi, tutti incamminati verso il sole che giganteggia all'orizzonte.

\section{Performatività e ripetizione}

Si è visto che l'immagine di Mussolini a cavallo poteva circolare nel mondo dei bambini e in quello degli adulti nelle stesse forme. Fotografie e cartoline erano i canali principali per questo tipo di osmosi, assicurata, oltre che da una possibile condivisione in famiglia degli oggetti in questione, dalla riproduzione fotografica dell'effigie mussoliniana nei libri scolastici: anche laddove i genitori non avessero collezionato fotografie o cartoline del Duce, cioè, i figli avrebbero comunque trovato le stesse immagini nei sussidiari. Oppure i libri di scuola e per ragazzi raccontavano di esperienze comuni ai bambini e agli adulti dell'Italia fascista, come potevano essere la visione di opere artistiche - abbiamo visto il caso degli affreschi - che ritraevano Mussolini in veste di condottiero o, molto più comuni, le periodiche leve fasciste con la rivista delle truppe condotta dal Duce in sella.

Quasi tutti i materiali che abbiamo analizzato risalgono agli anni Trenta o ai primi anni Quaranta. L'immagine del Duce a cavallo circolava già negli anni Venti, ma allora era in una doppia veste - di militare e borghese - che lo ritraevano soprattutto le cartoline e le fotografie; a partire dagli anni Trenta invece l'immagine del condottiero prevale e si diffonde attraverso i più vari canali; e parallelamente diventa sempre più forte il coinvolgimento dei minori nell'orizzonte militare su cui era incentrata la loro educazione ${ }^{34}$.

Come ha scritto Luisa Passerini, «il tema del vedere Mussolini non è certo una novità degli anni Trenta; $\mathrm{ma}$ in questo periodo diventa assillante e giunge ad articolarsi in una vera e propria tipologia. [...] C’è innanzitutto una visione che si avvale di qualsiasi immagine riprodotta: disegno, quadro, statua, sagoma, fotografia, usata spesso come aide-mémoire, capace di scatenare la fantasia ${ }^{35}$. Nel caso di Mussolini condottiero si è visto in

34. Cfr. ancora A. Gibelli, Il popolo bambino..., p. 33 : «Soprattutto a partire dagli anni Trenta, i minori sono intensamente coinvolti, strettamente coordinati, investiti da una straordinaria offensiva mediatica carica di suggestioni, incentrata sul tema [...] ossessivamente additato come modello insuperabile di virtù, di virilità, di successo : la proiezione plastica dell'infanzia sul futuro della forza combattente della nazione».

35. L. Passerini, Mussolini immaginario, p. 203. 
quale direzione fotografie, disegni, affreschi scatenassero la fantasia dei bambini: l'immagine del Duce a cavallo era associata alla fede nel fascismo, fatta di ardore, risolutezza, energia, forza, lotta per la patria, sentimento della vittoria, prestigio dell'Italia e dell'Impero; il risultato - ripetutamente narrato dalla penna degli adulti e, stando a quanto racconta Ciarlantini, effettivamente ottenuto - era che i bambini sviluppavano verso tutto questo un senso di emulazione e di partecipazione attiva.

"C’è poi » - scrive ancora Passerini - « un vedere in sogno, che ricorre anch'esso nei libri per ragazzi e che si apparenta alla fantasticheria a occhi aperti, ma che ha il potere di rendere più felici, configurandosi come un premio o una promessa ${ }^{36}$. Nel caso dell'effigie di Mussolini a cavallo si è visto che per i bambini non si trattava di una semplice contemplazione estatica paga di sé, premio al solo desiderio di vedere, ma si dovevano innescare delle reazioni ben precise: prima di tutto il senso di un rapporto personale con il Duce, con la sensazione di contribuire alla fede fascista (come nel caso del «bambino italiano» della poesia di Padellaro) o con il sentimento di un'investitura, di essere prescelti per un premio o una missione (Filo e Refe); e poi naturalmente c'era l'impegno in prima persona a spronare gli altri, a diffondere le idee del fascismo e a glorificare il regime (Filo e Refe nel deserto di Tripoli o Leonetto con il suo tamburo). La visione di Mussolini a cavallo, insomma, implicava un'azione; il vedere portava con sé il fare.

C'è una linea storiografica basata sul concetto di performativity che, mutuando da Judith Butler e dalla sua nozione di gender l'idea della costruzione dell'identità (nel suo caso di genere) come derivante dalla ripetizione di una serie di atti, vede il farsi dell'identità nazionale dei bambini cresciuti nei regimi totalitari in termini non solo di indottrinamento dall'alto, ma di agency e di performance, considerando cioè i minori non come soggetti necessariamente passivi ma anche attivi. Scrive Butler:

Gender is not a fact, the various acts of gender create the idea of gender, and without those acts, there would be no gender at all. Gender is, thus, a construction [...], the action of gender requires a performance that is repeated $[\ldots]$; gender is an identity tenuously constructed in time, instituted in an exterior space through a stylized repetition of acts ${ }^{37}$.

Studiando i bambini nella Cina di Mao alcuni studiosi come Terry E. Woronov e Xu Xu hanno lavorato a partire dalle stesse premesse. Scrive per esempio Woronov:

36. L. Passerini, Mussolini immaginario, p. 203.

37. J. Butler, Gender Trouble. Feminism and the Subversion of Identity, New York - Londra, Routledge, 1990, p. 140. 
The concept of performativity can also be usefully extended to help us think about the construction of children as national subjects, and the ways that nationalism is performed in some of the same ways that gender is. From this perspective, nationalism is understood as something children do, not something they acquire. It thus takes us beyond state-sponsored textbooks and propaganda, and focuses instead on children's daily lives to inquire how and where the nation is performed. These performances may include reading textbooks, but also a range of other daily activities ${ }^{38}$.

Se pensiamo al regime di Mussolini è possibile vedere nella stessa ottica anche la costruzione dell'identità fascista dei bambini italiani e capire ancora meglio il ruolo centrale che rivestiva in quel contesto l'immagine del Duce a cavallo. Come si è visto, questa immagine abitava l'orizzonte infantile a vari livelli : in primo luogo rimandava alle parate e alla leva fascista, cioè a un'esperienza che i bambini vivevano direttamente, anzi, a una performance a cui erano chiamati in prima persona quando Figli della Lupa, Balilla e Piccole Italiane sfilavano davanti al Duce a cavallo oppure alzavano il braccio al suo passaggio gridando «A noi! », proprio come facevano gli adulti. Era un'esperienza che si imprimeva nella memoria e che poi veniva rievocata nei temi e nei disegni in classe. Inoltre l'immagine di Mussolini a cavallo, che i bambini ritrovavano nei libri di scuola e nei racconti per ragazzi, rimandava a un orizzonte per così dire performativo anche quando era proposta attraverso la lettura: infatti l'azione dei piccoli protagonisti come Orazio, Leonetto, Filo e Refe, riproponeva - attraverso atti specifici come rifare i dipinti con Mussolini condottiero, suonare il tamburo al seguito del Duce, trovare il tesoro da lui indicato e istituire una milizia fascista tra gli indigeni - i valori che la propaganda di regime associava al fascismo e che sono stati già ricordati sopra: cioè energia, ardore, risolutezza, sentimento nazionale, missione imperiale, fede nel Capo.

Si è visto che Butler sottolinea nella costruzione del gender la componente della ripetizione. Secondo Butler, "As in other ritual social dramas, the action of gender requires a performance that is repeated. This repetition is at once a reenactment and reexperiencing of a set of meanings already socially established; and it is the mundane and ritualized form of their legitimation ${ }^{39}$. L'immagine di Mussolini a cavallo metteva in atto il

38. T.E. Woronov, «Performing the Nation: China's Children as Little Red Pioneers", Anthropological Quarterly, LXXX, 3, Summer 2007, p. 655. Si veda anche Xu Xu, «"Chairman Mao's Child": Sparkling Red Star and the Construction of Children in the Chinese Cultural Revolution", Children's Literature Association Quarterly, XXXVI, 4, Winter 2011, p. 381-409.

39. J. Butler, Gender Trouble..., p. 140. 
medesimo meccanismo di ripetizione: non solo era ripetuta e moltiplicata essa stessa attraverso fotografie, cartoline, disegni, filmati, racconti e poesie, ma la sua essenza era esattamente di tipo performativo, perché rimandava all'esperienza diretta delle parate - che i bambini ricordavano e ricreavano attivamente in temi e disegni - e alla proiezione fantastica di azioni eroiche e avventurose inserite tutte nel set of meanings del fascismo.

La nozione di performativity di Butler è già stata applicata al fascismo italiano. Anne Wingenter se ne è servita per analizzare le lettere che i bambini italiani in epoca fascista scrivevano al Duce e ha osservato: "As with youth group drills and parades or the rote memorization and recitation of fascist pledges, mottos and decalogues, children practiced the rules of the form, creating demonstrations of their belonging to a national community centered on the figure of the Duce ${ }^{40}$. Questo rimane vero anche nel nostro caso in cui i bambini erano sia produttori di testi e immagini con Mussolini a cavallo (si pensi di nuovo al libro di Mingozzi), sia soprattutto consumatori di un largo numero di favole e raffigurazioni incentrate sullo stesso personaggio proposto in chiave mitica. Certo, in questo secondo caso i racconti e le illustrazioni dei libri erano opera degli adulti, che ne controllavano strettamente il contenuto e lo rendevano pienamente conforme all'ideologia fascista; ma come dice Woronov le "performances may include reading textbooks"; e per altro il lavoro di fantasia fatto sui racconti scritti o attivato dalle figure correvano sempre in parallelo alle performances delle parate o dei campi Balilla.

Nell'ambito delle letture scolastiche o per ragazzi l'orizzonte performativo suscitato dall'immagine di Mussolini a cavallo prevedeva a volte che i bambini fossero al centro dell'azione o della scena: lo abbiamo visto con Orazio nel brano di Petrucci, con Filo e Refe nel libro di Colombini, con Leonetto su Monte Mario disegnato da Pullini. Ciò non è strano considerato il meccanismo di identificazione che si ricercava per ragioni educative in quei materiali o che era insito nel genere di scrittura. D'altra parte erano numerosi i casi in cui protagonista assoluto rimaneva Mussolini : basti pensare alla lettura «Il bimbo prodigioso (il Duce racconta)» che accompagnava la fotografia scattata all'ippodromo di villa Glori, ai quaderni stampati dalle cartiere Donzelli, al disegno di Pullini incorniciato nell'arco a tutto sesto e alla poesia di Petrucci sottostante; anche la poesia di Padellaro partiva dal bambino ma finiva con le parole e le promesse del Duce. Per non dire che la centralità e la superiorità di Mussolini erano sempre sottolineate $\mathrm{e}$ mai smentite durante le parate e le riviste militari: non solo la posizione

40. A. Wingenter, «"Vi saluto romanamente!". Self-narration and performance in children's letters to Mussolini», History of Education \& Children's Literature, VII, 1, 2012, p. 244. 
in sella lo poneva già in partenza più in alto degli altri - e ancora più in alto dunque dei bambini, di statura più bassa degli adulti - ma quando si muoveva il Duce era sempre primo, staccato dagli altri ufficiali a cavallo, oppure, se stava fermo in sella, si poneva non di rado su un rialzo di terra costruito appositamente per lui ${ }^{41}$. Non era dunque l'immaginazione dei fanciulli e delle "anime più semplici» a porre l'eroe-Duce «immediatamente su un piedestallo e in un'atmosfera di leggenda», come voleva Chiarini: era tutto il sistema di propaganda fascista che gli stava intorno e che costruiva la sua immagine di condottiero in maniera così studiata $\mathrm{e}$ capillare; erano gli adulti che creavano quel sistema e - con convinzione o anche subendolo - lo riassumevano così bene in quell'icona. I bambini non potevano che andare dietro a tutto questo.

\author{
Beatrice SICA \\ University College London
}

41. Cfr. il cinegiornale Luce con segnatura A0588 e le fotografie dell'archivio Luce con segnatura Aoooo1351, Aoooo1354, Aoooo9850, Aooo9851, A0021048, A0021049, A0021066, A0021073, Ao022111, Ao054536, tutti visibili sul sito internet dell'Archivio Luce, http://www.archivioluce. com/archivio (accesso effettuato il 26 maggio 2018); e inoltre le fotografie con segnatura RCB-F-014520-0000, RCB-F-015430-0000 e RCB-F-015431-0000 visibili nel sito internet dell'Archivio Alinari https://www.alinari.it/ (accesso effettuato il 26 maggio 2018). Il rialzo del terreno costruito per il Duce è anche ricordato da Mario Carli nel suo romanzo L'Italiano di Mussolini (Milano, Mondadori, 1930) che rievoca il quarto annuale della Marcia su Roma che fu celebrato con l'inaugurazione del nuovo Stadio Littoriale (oggi Dall'Ara) di Bologna il 31 ottobre 1926: «il Duce [...] apparve, solo, a cavallo, sul rialzo di terra costruito per lui, simile a un grande piedistallo da cui, vivente monumento equestre di se stesso, egli poteva tutti vedere e da tutti essere visto» (p. 199). 


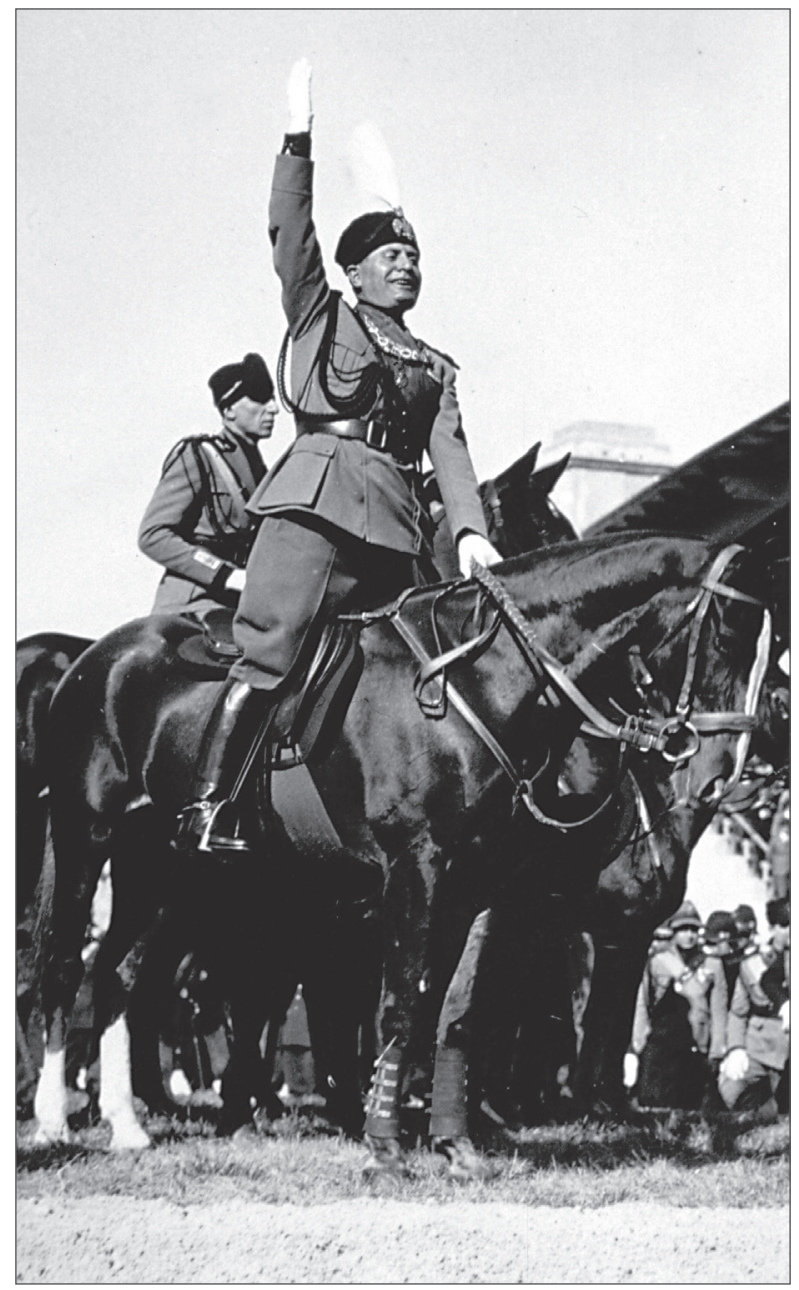

Fig. 1 - Eugenio Risi, ritratto a cavallo di Benito Mussolini. Archivi Alinari, Firenze. 


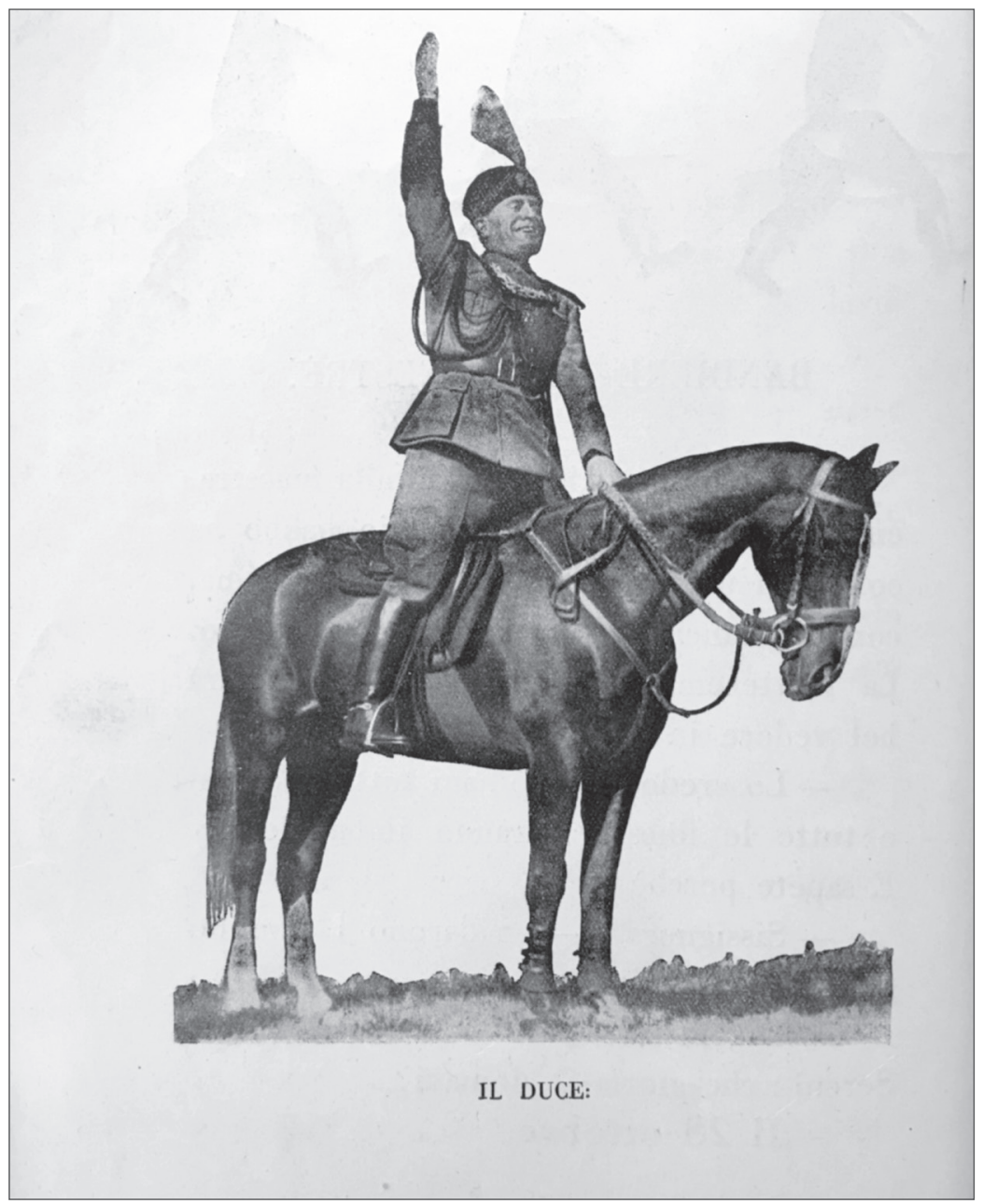

Fig. 2 - Fotografia (di Eugenio Risi) ritoccata di Mussolini a cavallo, da: Il libro della seconda classe, compilato dalla signora O. Quercia Tanzarella (Ornella), illustrato da M. Pompei, Palermo, Libreria dello Stato, 1930, p. 22. Per gentile concessione della Biblioteca di Storia Contemporanea «Alfredo Oriani ». 


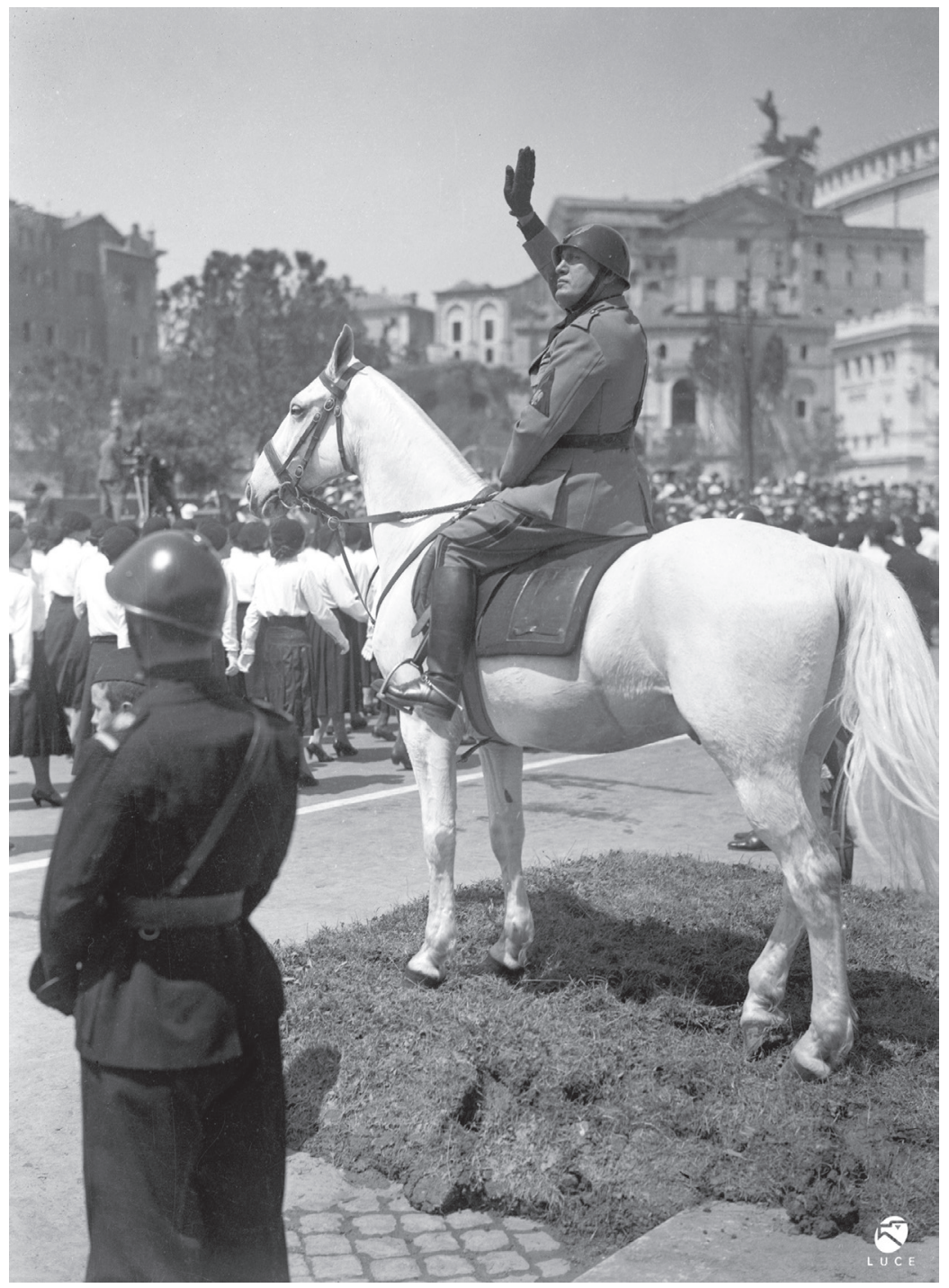

Fig. 3 - Mussolini a cavallo in via dell'Impero fa il saluto romano al passaggio delle Giovani Italiane. Roma, 24 maggio 1934, cerimonie dell'ottava leva fascista. Archivio Storico Istituto Luce. 


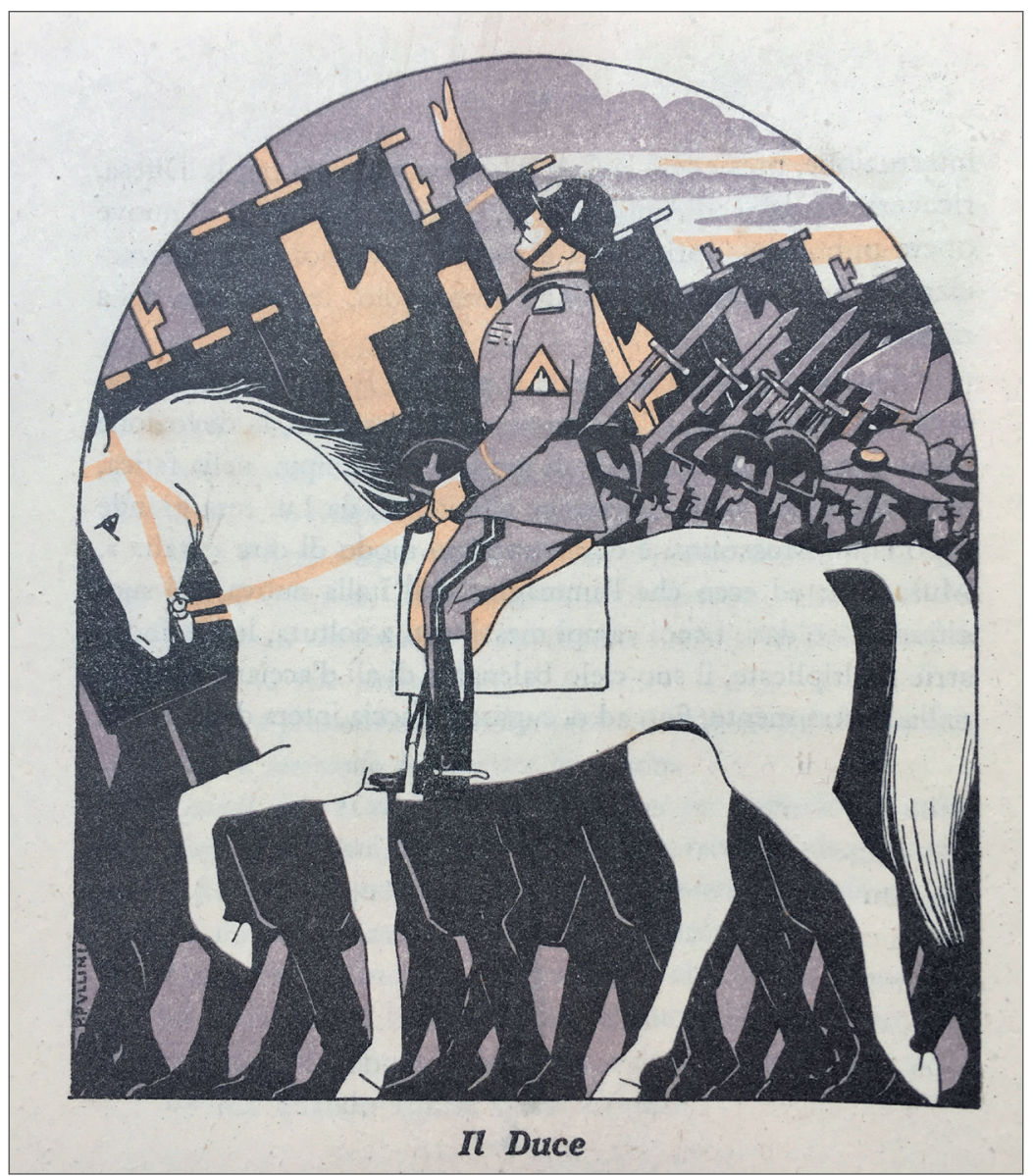

Fig. 4 - Pio Pullini, disegno di Mussolini a cavallo, da:

L'aratro e la spada. Letture per la terza classe dei centri rurali, testo di A. Petrucci, illustrazioni di P. Pullini, Roma,

Libreria dello Stato, 1941, p. 192.

Per gentile concessione degli eredi di Pio Pullini. 


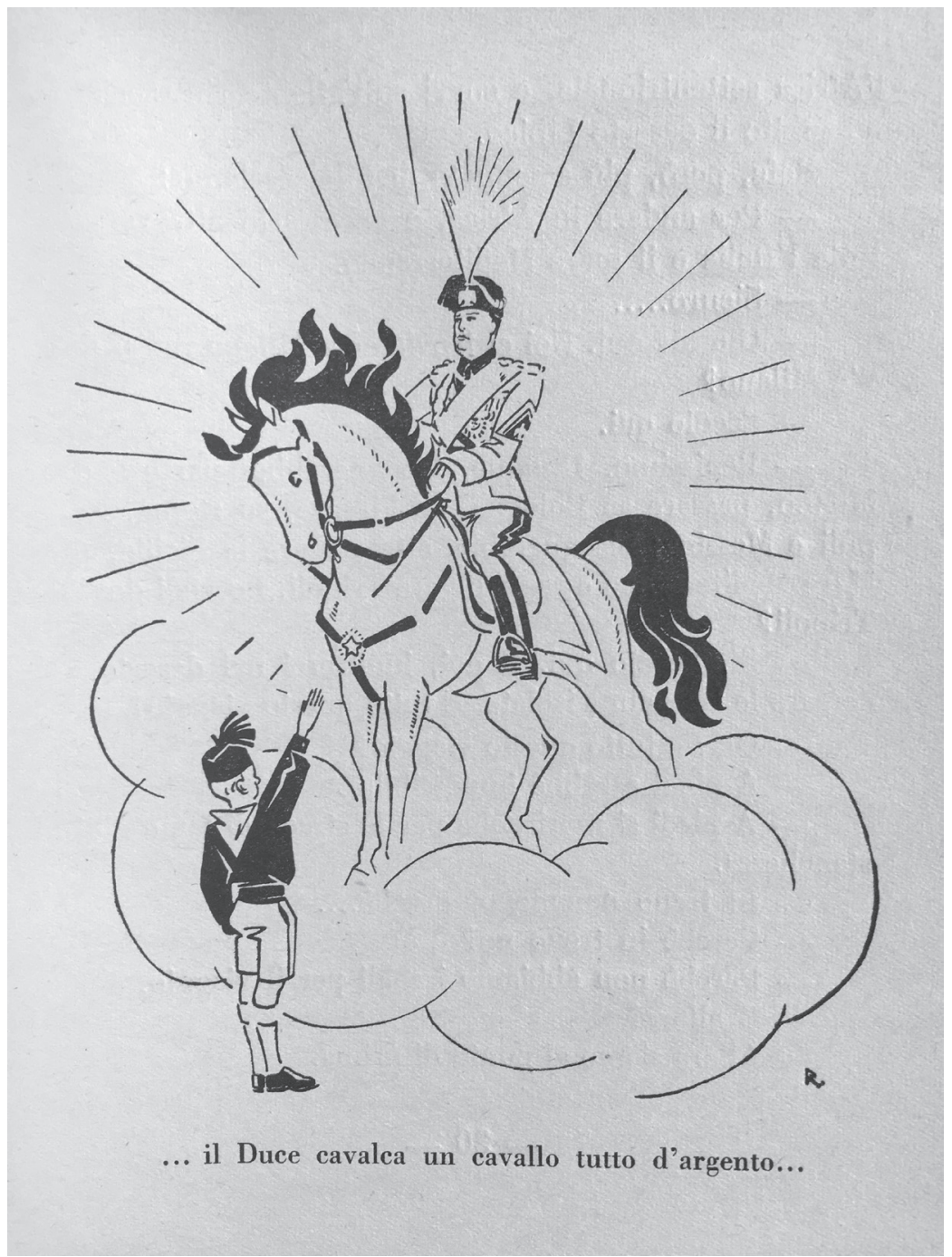

Fig. 5 - «R.», disegno di Mussolini a cavallo, da: P.A. Colombini, Filo e Refe alla scoperta di un tesoro, Milano, Istituto Tipografico Editoriale, 1934, p. 29. Collezione privata B.S. 


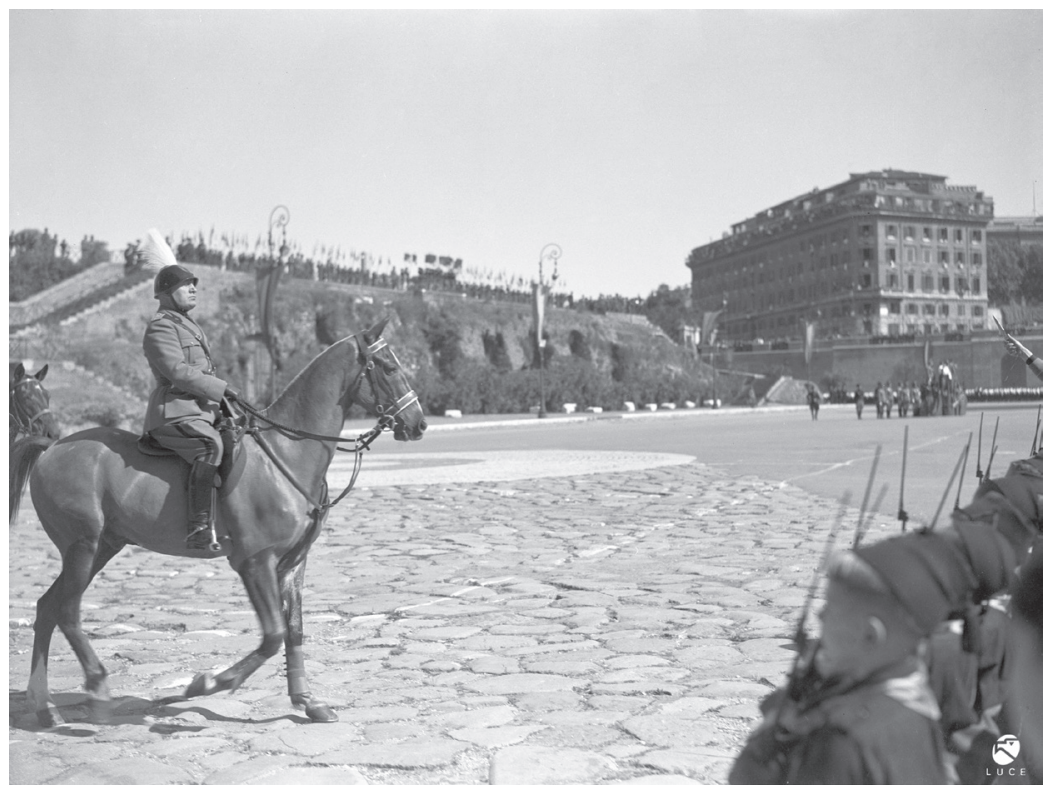

Fig. 6 - Mussolini a cavallo, in divisa di capo della Milizia con la piuma sull'elmetto, passa in rassegna dei balilla con il moschetto nei pressi del Colosseo. Archivio Storico Istituto Luce.

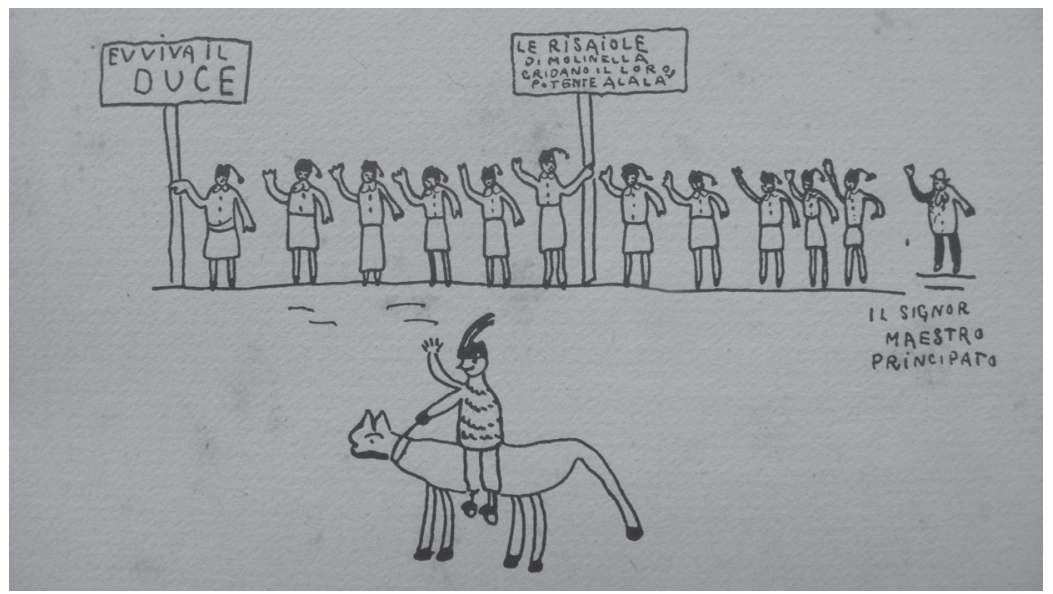

Fig. 7 - Disegno di una bambina di Molinella, da: D. Mingozzi, Mussolini visto dai ragazzi, con prefazione di A. Turati, San Casciano Val di Pesa (Firenze), Società Editrice Toscana, 1928, p. 43. Collezione privata B.S. 


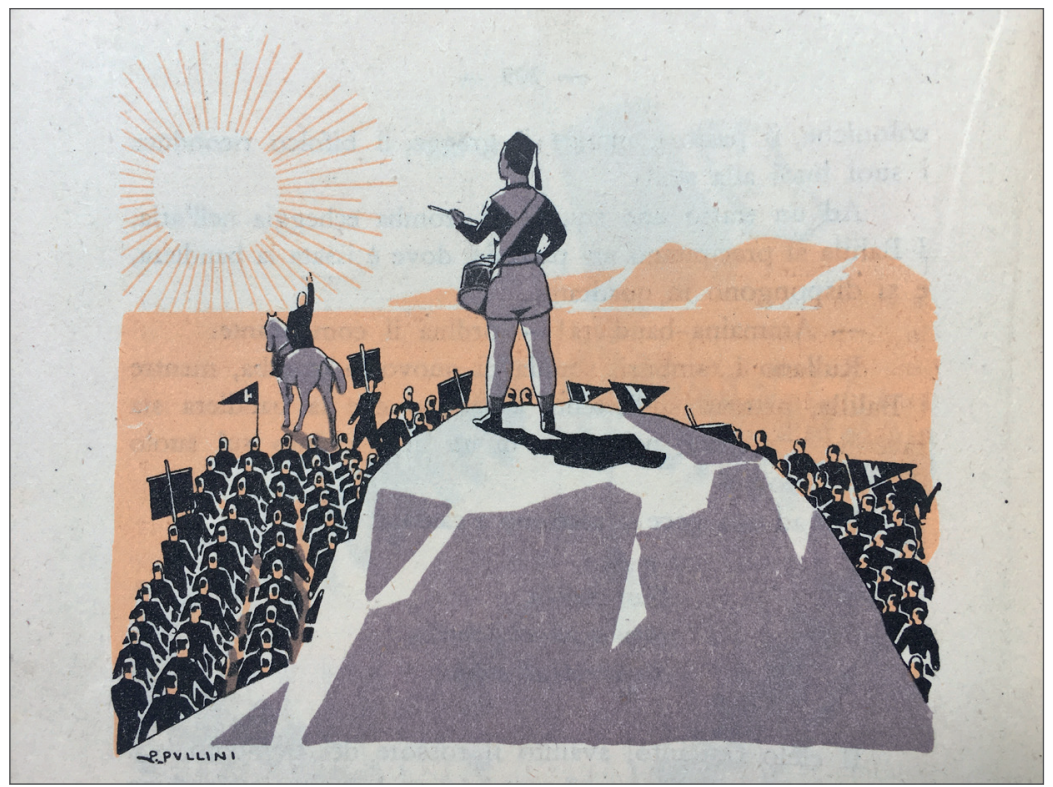

Fig. 8 - Pio Pullini, disegno di Leonetto sul Monte Sacro; sullo sfondo, Mussolini a cavallo. Da: L'aratro e la spada. Letture per la terza classe dei centri rurali, testo di A. Petrucci, illustrazioni di P. Pullini, Roma, Libreria dello Stato, 1941, p. 210.

Per gentile concessione degli eredi di Pio Pullini. 